\title{
Variação na estrutura diamétrica, composição florística e características sucessionais de fragmentos florestais da bacia do rio Guapiaçu (Guapimirim/Cachoeiras de Macacu, RJ, Brasil)
}

Ricardo Finotti ${ }^{1,4}$ Bruno Coutinho Kurtz ${ }^{2}$, Rui Cerqueira ${ }^{3}$ e Irene Garay ${ }^{1}$

Recebido em 14/06/2011. Aceito em 7/04/2012

\begin{abstract}
RESUMO
(Variação na estrutura diamétrica, composição florística e características sucessionais de fragmentos florestais da bacia do rio Guapiaçu (Guapimirim/Cachoeiras de Macacu, RJ, Brasil)). O presente trabalho compara a composição e estrutura de assembléias vegetais de fragmentos florestais, com diferentes características em relação ao tipo de propriedades rurais do entorno, tamanho e histórico de uso, com uma área localizada dentro de uma unidade de conservação (área controle), na bacia do rio Guapiaçu. Em cada fragmento foram delimitadas duas parcelas de 50 x 100m, na mesma orientação sudoeste, no interior das quais foram medidos os DAP (Diâmetro Acima do Peito) e altura de todos indivíduos com DAP $\geq 5 \mathrm{~cm}$ e identificados até o menor nível taxonômico possível. A densidade, área basal total, riqueza e diversidade foram calculados. As assembléias foram comparadas quanto aos seus valores diâmétricos e proporção de indivíduos em classes de diâmetro. Os parâmetros fitossociológicos foram calculados e as vinte espécies de maior cobertura foram selecionadas com vistas à comparação da composição específica e de suas caracteríticas sucessionais. Todas as assembleías estudadas apresentam alta riqueza e diversidade de espécies, sendo igualmente constatadas, em relação à área controle, diferenças significativas em pelo menos uma das caracteríticas analisadas. Todas apresentam uma menor proporção de indivíduos nas classes diâmetricas maiores e de espécies secundárias tardias que na área controle. No entanto, para algumas delas correspondem valores totais de diâmetro igual ou maiores que os da área controle por conta da maior proporção de indivíduos em classes diâmetricas intermediárias que parece contribuir mais para o aumento desses valores. De forma geral, há maior abundância de espécies pioneiras e secundárias iniciais nos fragmentos florestais, porém a proporção destas categorias sucessionais varia entre eles evidenciando diferenças dos estágios sucessionais em que se encontram. Estas diferenças poderiam estar relacionadas a certa variabilidade dos distúrbios identificados no entorno. Os resultados obtidos aqui sugerem que fragmentos de mata rodeados por pequenas propriedades encontram-se em estágios sucessionais mais inicias e sujeitos a distúrbios mais severos.
\end{abstract}

Palavras-chave: Fragmentos florestais, estágio sucessionais, classes de diâmetro, distúrbios, fitossociologia

\begin{abstract}
(Variations in structure, floristic composition and successional characteristics of forest fragments of the Guapiaçu river basin (Guapimirim/Cachoeiras de Macacu, RJ, Brazil)). This paper presents an analysis of the composition and structure of plant assemblages of forest fragments with different characteristics (in relation to the type of rural properties in the surrounding area, size and disturbance history) and an area located within a Conservation Unit (control area) in the Guapiaçu river basin. In each fragment, two plots of $50 \mathrm{x} 100 \mathrm{~m}$ were delimited to the South and West, the height and DBH of all trees with DBH $\geq$ $5 \mathrm{~cm}$ were measured, and the individuals were identified to the lowest possible taxonomic level. The density, basal area and diversity were calculated and the DAP values and proportion of individuals in the diameter classes were compared between the assemblages. The phytosociological parameters were also calculated and twenty species of higher coverage indices were selected to compare the species composition and their successional characteristics. All assemblages studied had high species richness and species diversity. However, when compared to the control, all of them had significant differences in at least one of analyzed characteristics. All had a lower proportion of individuals in the largest diameter class and late secondary species. However, these differences did not corresponded to differences in the comparisons of individual DAP values. Some assemblages had DAP values equal to or higher than the control area. In fact, within some fragments, the higher proportion of individuals in the intermediate diameter classes contributes more to the total basal area value. In general, there was a greater abundance of pioneer and early secondary species in the forest fragments, but the proportion of these successional groups varied between them, showing the subtle differences in the successional stages that these fragments are in. These differences could be related to some variability of the environmental disturbances identified. Based on the results, it was found that the forest fragments surrounded by small farms are in initial successional stages and have been subjected to severer disturbances.
\end{abstract}

Key words: forest fragments, successional stage, diameter classes, disturbances, phytosociology

\footnotetext{
Universidade Federal do Rio de Janeiro, Instituto de Biologia, Departamento de Botânica, Laboratório de Gestão da Biodiversidade, Rio de Janeiro, RJ, Brasil

Instituto de Pesquisas Jardim Botânico do Rio de Janeiro, Diretoria de Pesquisa Científica, Rio de Janeiro, RJ, Brasil

3 Universidade Federal do Rio de Janeiro, Instituto de Biologia, Departamento de Ecologia, Laboratório de Vertebrados, Rio de Janeiro, RJ, Brasil

4 Autor para correspondência: finotti@biologia.ufrj.br
} 


\section{Introdução}

Historicamente, as áreas florestadas das bacias fluviais da baixada fluminense sofreram intenso desmatamento por conta de diferentes ciclos econômicos desde o século XVI assim como outras áreas que possuíam quantidades significativas de Floresta Atlântica (Drummond 1997; Dean 2002). A ocupação do recôncavo da Baía de Guanabara deu-se a partir da plantação de cana-de-açucar, nos séculos XVI e XVII, que se expandiria por sobre os terrenos baixos e colinosos propícios ao estabelecimento de grandes propriedades (Amador 1997).

Nessa época, na bacia do rio Macacu, que engloba a sub-bacia do rio Guapiaçu, a floresta era derrubada tanto para dar lugar às plantações como para uso da madeira em construções e produção de lenha (Cabral 2006) e os rios da região eram importantes vias de escoamento de toda essa produção. Existem registros de ocupação humana e do uso dos recursos naturais da Bacia do rio Guapiaçu datados do século XVIII, período em que as florestas da região serviam como importantes fontes de fibras vegetais, sendo cenário de intensa atividade extrativista de madeira (Cabral 2006). Nesse século, também apareceram várias olarias que utilizavam a tabatinga, a argila e o barro como matéria prima e a madeira, como combustível para os fornos (Boher 2007). Na década de 1930, obras de retificação, canalização e dragagem foram realizadas nos principais rios da região, o que resultou na drenagem das áreas alagadas de baixada, possibilitando o adensamento populacional local (Cabral \& Fiszon 2004; Antunes et al. 2009). Portanto, as áreas que outrora eram ocupadas por florestas pantanosas foram sendo gradativamente substituídas por propriedades agrárias.

Atualmente, esta região é ocupada por pequenas propriedades rurais e de veraneio e por grandes propriedades ligadas à atividade agropecuária (Cabral \& Fiszon 2004). Contudo, pequenas porções de Floresta Atlântica, com áreas entre 19 e 200 ha, ainda são encontradas nos cumes dos morros (Freitas et al. 2005), sendo a maioria delas parte da reserva legal das propriedades do entorno.

A bacia do rio Guapiaçu possui remanescentes florestais importantes, com alta riqueza e diversidade de espécies em áreas localizadas no interior da Estação Ecológica Estadual do Paraíso (Kurtz \& Araújo 2000). Um estudo recente, realizado para a elaboração do plano de manejo da APA da bacia do rio Macacu (Rodrigues et al. 2009), demonstra que os remanescentes florestais localizados em propriedades privadas - que possuem diferentes tipos de entorno e que estão sob diferentes tipos de uso - possuem alta riqueza e diversidade de espécies e populações importantes de elementos representativos da flora da Floresta Atlântica de terras-baixa e submontana (senso IBGE 1992). Isto também é verdade para outros remanescentes florestais, de tamanho reduzido e com longo histórico de perturbação, localizados em bacias hidrográficas próximas e de mesma formação vegetacional (Pessoa \& Oliveira 2006; Rodrigues 2004; Carvalho et al. 2006). No entanto, esses e outros estudos que analisaram sistemas florestais em diferentes tempos de regeneração ou com diferentes históricos de perturbação mostram que florestas alteradas podem ter, em seu estágio de desenvolvimento tardio, características similares aos estágios sucessionais iniciais (Guedes-Bruni 1988, Oliveira-Filho et al. 1997; De Walt et al. 2003).

Comparados com florestas maduras, trechos de florestas em sucessão secundária são heterogêneos em vários aspectos (Santos et al. 2008). Características importantes podem ser consideradas como indicacoras das modificações ocasionadas por diversos impactos, tais como: a diminuição da área basal e densidade dos indivíduos nas classes diamétricas maiores, ocasionando uma diminuição na área basal total (Oliveira 2002; Gomes et al. 2004; Pessoa \& Oliveira 2006; Bobo et al. 2006), o aumento das espécies secundárias iniciais e pioneiras e a diminuição da riqueza e diversidade (Guariguata \& Ostertag 2001; Gomes et al. 2004; Villela et al. 2006). Porém, estas florestas nem sempre apresentam o padrão esperado de acordo com os tipos e intensidades de impactos previamente identificados. Parte desta dificuldade reside no fato de que esses impactos podem estar agindo em escalas de tempo e espaço múltiplas e são, portanto, de díficil determinação (Turner 1996; Dobson et al. 1997).

Sendo assim, o conhecimento da composição e estrutura e suas variações em assembléias vegetais são de vital importância para que se possa elaborar hipóteses sobre os processos ecológicos que contribuem para suas diferenças. O objetivo desse trabalho é comparar a estrutura e a composição florística do componente arbóreo de fragmentos de Floresta Atlântica de terras baixa e submontana de diferentes tamanhos e características, que estão inseridos no interior de propriedades rurais particulares na bacia do rio Guapiaçu, com a de uma área bem preservada localizada dentro da Estação Ecológica Estadual do Paraíso, na mesma região.

\section{Materiais e métodos}

\section{Área de estudo}

Os fragmentos de mata estudados situam-se na bacia do rio Guapiaçu, localizado nos municípios de Guapimirim e Cachoeiras de Macacu (22。 $21^{\prime}$ - $22^{\circ} 39^{\prime} \mathrm{S}$ e $\left.42^{\circ} 40^{\prime}-43^{\circ} 01^{\prime} \mathrm{W}\right)$ (Fig. 1). A bacia do rio Guapiaçu está compreendida dentro da bacia do rio Macacu, podendo ser considerada uma sub-bacia desta. O rio Macacu é o maior rio em extensão da área e deságua na baía de Guanabara (Boher 2007).

O clima da região é quente e úmido, sem uma estação marcadamente seca, correspondendo ao tipo Af na classificação de Koppen (1948). As normais climatológicas indicam uma temperatura média anual de $21,9^{\circ} \mathrm{C}$, sendo janeiro o mês mais quente $\left(25,3^{\circ} \mathrm{C}\right)$ e julho o mês mais frio $\left(17,9^{\circ} \mathrm{C}\right)$. A precipitação tem médias mensais que variam de $337,8 \mathrm{~mm}$ (fevereiro) a $59,3 \mathrm{~mm}$ (julho), com um total anual de $2050 \mathrm{~mm}$. O período que compreende os meses mais frios (maio a outubro) também é o de menor pluviosidade (Kurtz \& Araújo 2000). 


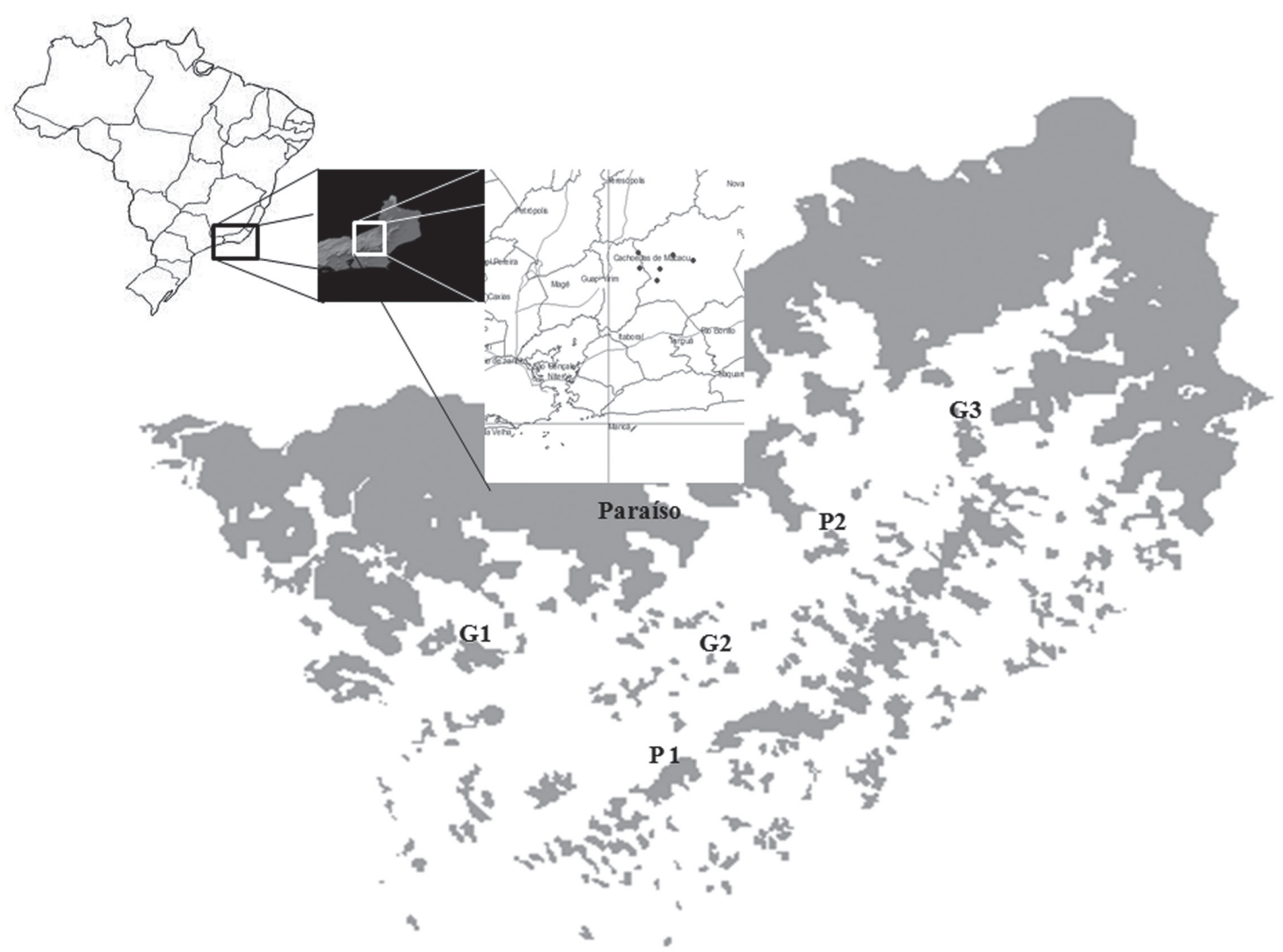

Figura 1. Localização dos fragmentos de mata estudados na bacia do rio Guapiaçú.

Do ponto de vista geológico, a bacia do rio Guapiaçu tem sua formação originada pelas atividades de magmatismo da era Cenozóica, pelos sedimentos fluvio-marinhos provenientes dos sequenciais continentais do Cenozóico, formando as Camadas Pré-Macacu e Formação Macacu, e pelos depósitos aluviais e coluviais do Holoceno. Estas camadas repousam sobre um embasamento cristalino do Pré-Cambriano (Amador 1997).

A área da bacia do rio Guapiaçú pertence ao domínio morfoclimático denominado "mares de morros florestados" (Ab'Saber 1971). Os morrotes do tipo meia-laranja possuem solos pertencentes à classe dos Latossolos Vermelho-Amarelo e a formação vegetacional característica é a Floresta Ombrófíla Densa Submontana (IBGE 1992).

Com o intuito de selecionar comunidades vegetais que pudessem apresentar diferenças estruturais e de composição específica, características como tamanho, grau de isolamento (distância em relação à área contínua), uso do solo no entorno e estrutura fundiária do entorno (pequenas ou grandes propriedades) foram levadas em consideração. Para isso, foi utilizado um mapa referência de fotos aéreas produzido pela Fundação CIDES (2003) (Escala 1:33500) e o trabalho de Cabral \& Fitzon (2004). As áreas selecionadas foram visitadas in loco para verificar a acessibilidade, obter permissão dos proprietários para o trabalho e tentar obter detalhes de seu histórico de uso. Desta forma, foram selecionados cinco fragmentos de mata, que foram classificados em $\mathrm{P} 1$ e $\mathrm{P} 2$, por estarem cercados por pequenas propriedades rurais, e G1, G2 e G3, por estarem inseridos dentro de uma grande propriedade rural. Foi igualmente selecionada uma área controle, com características topográficas e altitude semelhantes, localizada na Estação Ecológica Estadual do Paraíso (Paraíso) (Fig. 1 e Tab. 1).

No estado do Rio de Janeiro, as encostas voltadas para o quadrante Norte são mais quentes e secas que as encostas voltadas para o quadrante Sul (Figueiredo 1950; Aragão 1961; Bierkeland 1974; Turton \& Frieburger 1997). Oliveira et al. (1995) demonstraram que existem diferenças significativas entre estas encostas com relação à composição específica da comunidade arbórea e que estas diferenças estão associadas às diferenças nas variáveis abióticas. Portanto, para fins de comparação das assembléias vegetais aqui estudadas, apenas as vertentes voltadas para Sudoeste foram amostradas, pois são as vertentes mais úmidas (Oliveira et al. 1995).

Em cada fragmento, foram implantadas 2 parcelas de $50 \times 100$ m, uma no sentido Norte-Sul e outra no sentido Leste-Oeste, totalizando 1 ha de área amostral. O ínicio das 
Tabela 1. Características dos fragmentos florestais selecionados. Coordenadas geográficas, Tipos de propriedade do entorno, área (em hectares) e observações sobre o histórico e outras características consideradas relevantes.

\begin{tabular}{|c|c|c|c|}
\hline Áreas & Coordenadas & área (ha) & Observações \\
\hline $\begin{array}{l}\text { P1 } \\
\text { (Assentamento do INCRA) }\end{array}$ & $\begin{array}{l}22^{\circ} 34^{\prime} 38.4^{\prime \prime} \mathrm{S} \\
42^{\circ} 50^{\prime} 45^{\prime \prime} \mathrm{W}\end{array}$ & 157,36 & $\begin{array}{l}\text { Cercada de pequenos assentamentos realizados pelo INCRA na década de 1960, possui evidências } \\
\text { de eventos de fogo acontecidos há cerca de } 5 \text { anos atrás e corte de madeira em seu interior. Até } \\
\text { meados da década de } 70 \text { este fragmento estava conectado a uma extensa área de mata contínua } \\
\text { que sofreu intensa fragmentação formando uma grande quantidade de fragmentos pequenos } \\
\text { além deste }\end{array}$ \\
\hline $\begin{array}{l}\text { P2 } \\
\text { (próximo a Fazenda Recanto) }\end{array}$ & $\begin{array}{l}22^{\circ} 29^{\prime} 59,1^{\prime \prime} \mathrm{S} \\
42^{\circ} 47^{\prime} 43^{\prime \prime} \mathrm{W}\end{array}$ & 68,25 & $\begin{array}{l}\text { Possui muitas pequenas propriedades e uma propriedade considerada média em seu entorno. } \\
\text { Possui evidências de corte de madeira em seu interior. Este fragmento sofre intensa degradação } \\
\text { e perda da área florestada desde a década de } 70\end{array}$ \\
\hline $\begin{array}{l}\text { G1 } \\
\text { (Fazenda do Sr. Renato } \\
\text { Cozzolino) }\end{array}$ & $\begin{array}{l}22^{\circ} 32^{\prime} 18^{\prime \prime} \mathrm{S} \\
42^{\circ} 54^{\prime} 00^{\prime \prime} \mathrm{W}\end{array}$ & 119,58 & $\begin{array}{l}\text { Fragmento situado dentro de uma grande propriedade rural. Seu entorno é cercado por } \\
\text { plantações de aipim. Área com evidências de intenso corte de madeira para a utilização em } \\
\text { fornos de olaria para a confecção de telhas nos anos de } 1970 \text { e } 1980 \text {. }\end{array}$ \\
\hline $\begin{array}{l}\text { G2 } \\
\text { (Areal Santa Helena) }\end{array}$ & $\begin{array}{l}22^{\circ} 32^{\prime} 30.6^{\prime \prime} \mathrm{S} \\
42^{\circ} 50^{\prime} 15^{\prime \prime} \mathrm{W}\end{array}$ & 15,74 & $\begin{array}{l}\text { Área situada dentro de uma propriedade de extração de areia e onde se pratica pecuária } \\
\text { extensiva. Evidência de corte de madeira }\end{array}$ \\
\hline $\begin{array}{l}\text { G3 } \\
\text { (Fragmento do Sr. Odinho) }\end{array}$ & $\begin{array}{l}22^{\circ} 30^{\prime} 54^{\prime \prime} \mathrm{S} \\
42^{\circ} 43^{\prime} 49^{\prime \prime} \mathrm{W}\end{array}$ & 99,58 & $\begin{array}{l}\text { Fragmento situado no interior de uma grande propriedade rural, próximo à área de mata } \\
\text { contínua, rodeado por estradas de terra. Foram observadas poucas evidências de corte em seu } \\
\text { interior e a existência de plantações de espécies frutíferas exóticas tais como banana (Musa } \\
\text { paradisíaca L.) e alguns indivíduos de jaca (Artocarpus heterophyllus Lam.) }\end{array}$ \\
\hline $\begin{array}{l}\text { Paraíso } \\
\text { (Estação Ecológica Paraíso) }\end{array}$ & $\begin{array}{c}22^{\circ} 29^{\prime} 36^{\prime \prime S} \\
42^{\circ} 54^{\prime} 19.95^{\prime \prime} \mathrm{W}\end{array}$ & 53372,5 & $\begin{array}{l}\text { A área amostrada está localizada próxima à entrada da U.C. na localidade de Paraíso (Guapi- } \\
\text { Mirim-RJ). Em seu entorno imediato podem ser observadas pequenas plantações de coco } \\
\text { (Cocos nucifera L.) e algumas residências. Esta área está sob proteção, desde a década de 1930, da } \\
\text { Companhia Estadual de Águas e Esgoto (CEDAE) do Rio de Janeiro e, desde 1960, do Centro de } \\
\text { Primatologia e da Estação Ecológica do Paraíso }\end{array}$ \\
\hline
\end{tabular}

parcelas foi delimitado a 20 metros da borda do fragmento para evitar variações mais drásticas na composição e nos parâmetros estruturais relacionados ao efeito de borda. Em cada parcela, foram incluídos todos os indivíduos vivos com diâmetro do tronco a $1,30 \mathrm{~m}$ do solo (DAP) maior ou igual a $5 \mathrm{~cm}$. A altura de cada indivíduo foi estimada visualmente. Astrocaryum aculeatissimum (Schott) Burret e Cyathea corcovadensis (Raddi) Domin tiveram seus diâmetros medidos à altura do solo. Os indivíduos que apresentaram ramificações (rebrotos) foram medidos logo abaixo destas, muitas vezes pouco acima da superfície do solo. Os indivíduos foram marcados e material vegetativo e/ ou reprodutivo, quando disponível, foi coletado. As espécies foram identificadas até o menor nível taxonômico possível e o material depositado nos herbários do Jardim Botânico do Rio de Janeiro (RB) e do Departamento de Botânica do Instituto de Biologia da Universidade Federal do Rio de Janeiro (RFA).

\section{Análise dos dados}

Foram calculadas a média e desvio padrão dos diâmetros e alturas dos indivíduos, o número de famílias, a riqueza, a equitabilidade de Pielou (J) e o índice de diversidade de Shannon (H') para cada um dos fragmentos estudados (Magurran 1988).

Com base nos valores individuais de DAP, os fragmentos foram comparados pela análise de variância não-paramétrica de Kruskall-Wallis (H), utilizando-se a posteriori o teste $\mathrm{Z}$ para comparações múltiplas. Os cálculos foram feitos no software Statistica 7.0 (Statsoft INC.).
Os indivíduos de cada fragmento foram agrupados em classes de DAP, a saber: $0,05-0,10 \mathrm{~m}, 0,10-0,15 \mathrm{~m}, 0,15-$ $0,20 \mathrm{~m}, 0,20-0,25 \mathrm{~m}, 0,25-0,30 \mathrm{~m}$ e $>0,30 \mathrm{~m}$, sendo calculada a proporção de indivíduos em cada uma das classes (n de cada classe/ $\mathrm{n}$ total da amostra por fragmento), Estas proporções foram comparadas par-a-par entre os fragmentos através do teste Z para comparação de proporções (Rodrigues 1993). $\mathrm{O}$ valor de $\mathrm{Z}$ foi calculado através da divisão das diferenças das proporções pela diferença dos seus respectivos desvios-padrões. O nível de significância do valor de Z foi obtido numa tabela de proporções da curva normal.

O número de indivíduos de cada espécie foi utilizado em uma Análise de Correspondência Distendida (Detrended Correspondence Analysis - DCA-) (Hill \& Gauch 1980). Para isto, as espécies encontradas em apenas 1 fragmento florestal foram excluídas.

Para as espécies de cada fragmento, foram calculados os parâmetros densidade relativa (DR), dominância relativa (DoR) e o valor de cobertura (VC) (Mueller-Dombois \& Ellenberg 1974). As vinte espécies de maior VC de cada fragmento foram agrupadas em um dentre três grupos sucessionais, de acordo com a classificação de Carvalho et al. (2007; 2008) e Santos et al. (2008), seguindo os critérios de Gandolfi et al. (1995), a saber: Pioneiras (PI), Secundárias Iniciais (SI) e Secundárias Tardias (ST). Rodrigues (2004) ressalta que a análise da composição das espécies vegetais de maior valor de importância ou cobertura pode ser um bom indicador dos fatores que afetam uma assembléia localmente. Por isso, e pela dificuldade de realizar esta classificação sucessional para todas as espécies, esta abordagem foi utilizada. A 
proporção de espécies em cada um dos grupos sucessionais para cada fragmento foi então calculada. As proporções de cada grupo sucessional correspondentes aos diferentes fragmentos foram comparadas pelo teste Z para comparação de proporções, descrito acima (Rodrigues 1993).

\section{Resultados}

Os valores de riqueza variaram de 134 no fragmento $\mathrm{P} 1$ a 181 em Paraíso e os de diversidade variaram de 3,28 no fragmento G1 a 4,33 em Paraíso. Os valores de densidade variaram de $1020 \mathrm{ind} / \mathrm{ha}$ no fragmento $\mathrm{P} 1 \mathrm{a} 1410 \mathrm{ind} / \mathrm{ha}$ no fragmento G1 e a área basal variou de $12,61 \mathrm{~m}^{2} / \mathrm{ha}$ no fragmento P1 a 27,92 $\mathrm{m}^{2} /$ ha no fragmento G3 (Tab. 2).

Os valores de DAP foram significativamente diferentes entre os fragmentos florestais estudados $(n=6962, \mathrm{H}=$ $166,68, \mathrm{p}=0.0001$ ), sendo os valores de DAP de P1 significativamente menores que todos os outros fragmentos com exceção de G1 (Tab. 2 e 3). G1 também apresentou valores de DAP significativamente menores que P2, G2 e G3 e os valores de DAP de G3 foram significativamente maiores que todos os outros fragmentos (Tab. 3).

Estas diferenças se devem a existência de uma maior proporção de indivíduos na menor classe de diâmetro $(0,05-$ $0,1 \mathrm{~m})$ de $\mathrm{P} 1$ em relação à todos os fragmentos $(\mathrm{P} 1 \times \mathrm{P} 2$, $\mathrm{Z}=3,02, \mathrm{p}=0,01 ; \mathrm{P} 1 \times \mathrm{x} 2, \mathrm{Z}=1,82, \mathrm{p}=0,04 ; \mathrm{P} 1 \times \mathrm{G} 3, \mathrm{Z}=5,10$, $\mathrm{p}=0,005$; P1 x Paraíso, $Z=1,85, \mathrm{p}=0,038)$, com exceção de G1 ( $\mathrm{Z}=0,62$, $\mathrm{p}=0.4)$ (Fig. 2). Nesta classe, G1 também apresentou uma proporção de indivíduos significativamente menor em relação a todos os outros fragmentos ( $G 1 \times$ P2, $\mathrm{Z}=2,62, \mathrm{p}=0,02 ; \mathrm{G} 1 \times \mathrm{G} 2, \mathrm{Z}=1,33, \mathrm{p}=0,45 ; \mathrm{G} 1 \times \mathrm{G} 3, \mathrm{Z}=5,77$, $\mathrm{p}=0,003$; G1 x Paraíso, $Z=1,36, \mathrm{p}=0,038)$. P1 e G1 também apresentaram menor proporção de indivíduos na classe de maior diâmetro $(>0.3 \mathrm{~m})$ em relação a Paraíso ( $\mathrm{P} 1$ x Paraíso, $\mathrm{Z}=1,85, \mathrm{p}=0,038$ e G1 x Paraíso, $\mathrm{Z}=1,48, \mathrm{p}=0,043)$. Paraíso também apresentou uma menor proporção em relação a todos os fragmentos na classe de DAP 0.1-0.15m (P1 x Paraíso, $\mathrm{Z}=1,46, \mathrm{p}=0,043 ; \mathrm{P} 2 \times$ Paraíso, $\mathrm{Z}=1,99, \mathrm{p}=0,04, \mathrm{G} 1 \times$ Paraíso, $\mathrm{Z}=1,51, \mathrm{p}=0,04, \mathrm{G} 2 \times$ Paraíso, $\mathrm{Z}=1,40, \mathrm{p}=0,045$, G3 $\times$ Paraíso, $\mathrm{Z}=1,98, \mathrm{p}=0,035)$. $\mathrm{G} 3$ apresentou uma maior proporção de indivíduos em uma classe intermediária $(0,15-0,2 \mathrm{~m})$ em relação à todos os outros fragmentos (G3 x P1. Z=1,40, p=0,04, G3 x $\mathrm{G} 1, \mathrm{Z}=1,75, \mathrm{p}=0,03, \mathrm{G} 3 \times \mathrm{G} 2, \mathrm{Z}=2,13, \mathrm{p}=0,025$, G3 x Paraíso, $\mathrm{Z}=1,40, \mathrm{p}=0,04$ ), com exceção de P2 (Fig. 2).

Considerando todos os fragmentos, foram medidos 6962 indivíduos, pertencentes a 57 Famílias e 399 morfo-espécies. Dentre estas, $312(78,20 \%)$ foram identificadas até o nível específico, 75 (18,80\%) foram identificados até gênero e apenas 12 (3\%) não possuem nenhuma identificação. Quase a metade das espécies coletadas (45\%, 180 espécies) foi encontrada em apenas um fragmento florestal. Algumas das espécies que estão presentes em todas as comunidades aqui estudadas são classificadas como "Vulnerável" na listagem de espécies da IUCN, como é o caso de Sorocea guilleminiana Gaudich. e Mollinedea glabra (Spreng.) Perkins. Outras espécies também classificadas como vulnerável ou raras pelo IBAMA, tais como: Astromuim fraxinifolium Schott, Melanoxylum braúna Schott e Brosimum glaziovii Taub., são encontradas em baixas densidades em alguns desses fragmentos.

Por meio dos resultados da Análise de Correspondência Distendida (DCA) pode se perceber a singularidade destes fragmentos florestais no que diz respeito à composição específica. Os dois primeiros eixos da DCA explicam 26,73\% e $21,48 \%$ da variância total, respectivamente. A posição dos fragmentos nesses eixos mostra que os fragmentos de mata P1 e Paraíso estão associados aos extremos do eixo 1 e o fragmento G1 ocupa uma posição intermediária nesse eixo. Os fragmentos P2, G2 e G3 ocupam os extremos do eixo 2 (Fig. 3).

As dez espécies com maiores postos, associadas ao extremo do eixo 1 em que se encontra Paraíso, são consideradas, na sua maioria, espécies secundárias tardias; são elas: Cedrela odorata L., Guarea guidonia (L.) Sleumer, Ficus pulchella Schott, Algernonia cf. riedelii (Müll.Arg.) G.L.Webster, Mollinedia glabra (Spreng.) Perkins, Allophylus petiolulatus Radlk., Tabernaemontana laeta Mart, Amaioua intermedia Mart. ex Schult. \& Schult.f., Andradaea floribunda Allemão e Marlierea excoriata Mart. Aquelas associadas ao outro extremo, onde se encontra $\mathrm{P} 1$, são consideradas como tendo características de espécies pioneiras; são elas: Chamaecrista ensiformis (Vell.) H.S.Irwin \& Barneby, Stryphnodendron polyphyllum Mart., Inga lanceifolia Benth., Miconia prasina (Sw.)DC., Miconia cinnamomifolia (DC.) Naudin, Eugenia villaenovae Kiaersk., Maytenus communis Reissek, Pogonophora schomburgkiana Miers ex Benth, Tibouchina granulosa (Desr.) Cogn., Cecropia hololeuca Miq. e Jacaranda macrantha Cham. (Gandolfi et al. 1995).

Em relação ao segundo eixo fatorial, as espécies que estão associadas aos dois extremos desse eixo são consideradas espécies secundárias iniciais. As espécies associadas ao extremo deste eixo, onde se encontra P2, são: Asteraceae sp1, Eugenia brasiliensis Lam., Piptadenia gonoacantha (Mart) J.F. Macbr., Oxandra nitida R.E.Fr., Astronium graveolens Jacq., Sapium cf. glandulosum (L.) Morong, Myrcia pubipetala Miq., Solanum pseudoquina A.St.-Hil., Sloanea hirsuta (Schott) Planch ex. Benth e Brosimum lactescens (S.Moore) C.C. Berg, enquanto que no outro extremo, onde encontram os fragmentos G2 e G3 as espécies são: Cordia sellowiana Cham., Handroanthus chrysotrichus (Mart. ex DC.) Mattos, Andira fraxinifolia Benth., Psychotria carthagenensis Jacq., Euterpe edulis Mart., Psychotria glaziovii Müll.Arg., Schefflera calva (Cham.) Frodin \& Fiaschi, Protium widgrenii Engl., Helicostylis tomentosa (Poepp. \& Endl.) Rusby e Pera heteranthera (Schrank) I.M.Johnst.

As vinte espécies de maior VC de cada fragmento representam mais de $50 \%$ do valor de cobertura $(\mathrm{P} 1=69,23 \%$, P2: 58.84\%, G1: 57,70\%, G2: 68,64\%, G3: 65,59\%, Paraíso: 55,90\%). Apenas uma espécie, Astrocaryum aculeatissimum (Schott.) Burret, foi considerada de maior VC em todas as assembléias estudadas, sendo a primeira ou segunda espécie 
Tabela 2. Média \pm desvio padrão de DAP e altura e valores de densidade, área basal, número de famílias, riqueza, índice de equitabilidade de Pielou (J) e índice de diversidade de Shannon-Wiever $(\mathrm{H})$, para os fragmentos estudados.

\begin{tabular}{|c|c|c|c|c|c|c|}
\hline & $\mathrm{P} 1$ & $\mathrm{P} 2$ & G1 & G2 & G3 & Paraíso \\
\hline $\mathrm{DAP}(\mathrm{m})$ & $0,08 \pm 0,06$ & $0,09 \pm 0,09$ & $0,08 \pm 0,08$ & $0,10 \pm 0,09$ & $0,10 \pm 0,10$ & $0,09 \pm 0,11$ \\
\hline Altura (m) & $6 \pm 3,4$ & $6 \pm 4,5$ & $8 \pm 4,9$ & $7 \pm 5,3$ & $8 \pm 5,6$ & $7 \pm 4,8$ \\
\hline Densidade (ind/ha) & 1020 & 1095 & 1410 & 1112 & 1204 & 1128 \\
\hline Área basal $\left(\mathrm{m}^{2} / \mathrm{ha}\right)$ & 12,61 & 20,82 & 20,54 & 20,77 & 27,92 & 26,35 \\
\hline Famílias & 35 & 41 & 35 & 41 & 42 & 40 \\
\hline Riqueza & 134 & 168 & 137 & 163 & 161 & 181 \\
\hline $\mathrm{J}$ & 0,81 & 0,76 & 0,67 & 0,82 & 0,72 & 0,83 \\
\hline Diversidade $(\mathrm{H})$ & 3,97 & 3,9 & 3,28 & 4,16 & 3,65 & 4,33 \\
\hline
\end{tabular}

Tabela 3. Valores de $\mathrm{Z}$ correspondentes as comparações entre as comunidades com relação ao DAP em metros. * $a \leq 5 \%$.

\begin{tabular}{cccccc}
\hline & P2 & G1 & G2 & G3 & PARAÍSO \\
\hline P1 & $6,77^{*}$ & 2,54 & $7,57^{*}$ & $11,15^{*}$ & $4,08^{*}$ \\
P2 & & $4,72^{*}$ & 0,79 & $4,31^{*}$ & 2,79 \\
G1 & & & $5,58^{*}$ & $9,43^{*}$ & 1,79 \\
G2 & & & & $3,52^{*}$ & 2,60 \\
G3 & & & & & $7,20^{*}$ \\
\hline
\end{tabular}

em maior valor desse parâmetro em todas elas. Aparisthmium cordatum (A. Juss.) Baill. e Guarea macrophyla Vahl. estão entre as espécies de maior VC em 5 das 6 comunidades estudadas. A primeira não está entre as vinte primeiras espécies de maior VC em Paraíso e a segunda no fragmento florestal P1 (Tab. 4).

A análise das espécies de maior VC classificadas de acordo com o grupo sucessional mostra que, com exceção de P1 e Paraíso, as assembléias estudadas têm uma proporção maior de espécies classificadas como secundárias iniciais (Fig. 4). A proporção de espécies classificadas como secundárias tardias é significativamente maior em Paraíso quando comparadas às outras comunidades arbóreas (Paraíso x P1: $\mathrm{Z}=3,46, \mathrm{p}=0,02$; Paraíso x P2 e $\mathrm{G} 1, \mathrm{Z}=1,65, \mathrm{p}=0,03$; Paraíso $\mathrm{x}$ G2 e G3, $\mathrm{Z}=1,30, \mathrm{p}=0,035$ ) e a proporção de espécies classificadas como pioneiras é significativamente maior em $\mathrm{P} 1$ em relação a todas as outras comunidades $(\mathrm{P} 1 \times \mathrm{P} 2, \mathrm{Z}=2,03, \mathrm{p}=0,038 ; \mathrm{P} 1 \times \mathrm{G} 1$, $Z=2,92, p=0,35 ; P 1 \times G 2, Z=3,92, p=0,18 ; P 1 \times G 3, Z=3,46$, $\mathrm{p}=0,15$ e $\mathrm{P} 1 \mathrm{x}$ Paraíso, $\mathrm{Z}=4,12, \mathrm{p}=0,01)$. Os fragmentos G1 e G3 apresentaram uma proporção significativamente maior de espécies classificadas como secundárias iniciais quando comparada a P1 e a Paraíso (para as quatro comparações $Z=1,30, p=0,04)$. $P 2$ apresentou uma proporção significativamente maior de espécies pioneiras quando comparada a Paraíso $(Z=1,85, p=0,03)$, G2 e G3 ( $Z=1,27$, $\mathrm{p}=0,045$ para as duas comparações). P1 apresentou proporção significativamente menor de espécies classificadas como secundárias tardias em relação a todas as outras comunidades (P1 x P2 e G1, Z=1,63. p=0,04; P1 x G2 e G3, $\mathrm{Z}=1,98, \mathrm{p}=0,02$ ) (Fig. 4).

\section{Discussão}

O fato das assembléias vegetais da bacia do rio Guapiaçu, não só possuírem valores de riqueza e diversidade similares a outras da mesma bacia hidrográfica ou de regiões próximas (Kurtz \& Araújo 2000, Rodrigues 2004, Pessoa \& Oliveira, 2006), mas também apresentarem espécies raras e/ou ameaçadas de extinção demonstra a importância desta bacia do ponto de vista da conservação das populações vegetais. A baixa similaridade entre os fragmentos florestais, dadas a alta proporção de espécies exclusivas a um único fragmento florestal e as diferenças na composição de espécies e suas respectivas abundâncias nas assembléias estudadas, é uma característica comum a remanescentes florestais de formação terras-baixa e submontana (Oliveira-filho \& Fontes 2000; Borém \& Ramos 2001; Scudeler et al. 2001; Rodrigues 2004; Peixoto et al., 2004; Pereira et al. 2007) e reforça a importância da preservação de cada remanescente florestal na manutenção do status da biodiversidade (Pellens et al. 2009). No entanto, todos os fragmentos florestais inseridos dentro das propriedades particulares demonstram diferenças em alguma característica de composição e de estrutura quando comparadas à área controle, o que evidencia que esses fragmentos são compostos, na sua grande maioria, por fragmentos florestais secundários, característica esta também comum de áreas fragmentadas (Murcia 1995).

Uma das alterações observadas em florestas secundárias é a diminuição da área basal e da densidade de indivíduos nas classes de diâmetro maiores, ocasionando uma diminuição na área basal total (Oliveira 2002; Gomes et al. 2004; Pessoa \& Oliveira 2006; Bobo et al. 2006). Analisando fragmentos florestais no nordeste do Brasil, Oliveira et al. (2008) demonstram que existe um empobrecimento do componente arbóreo de maior diâmetro e redução significativa dos valores de DAP nas bordas de fragmentos florestais e em fragmentos 


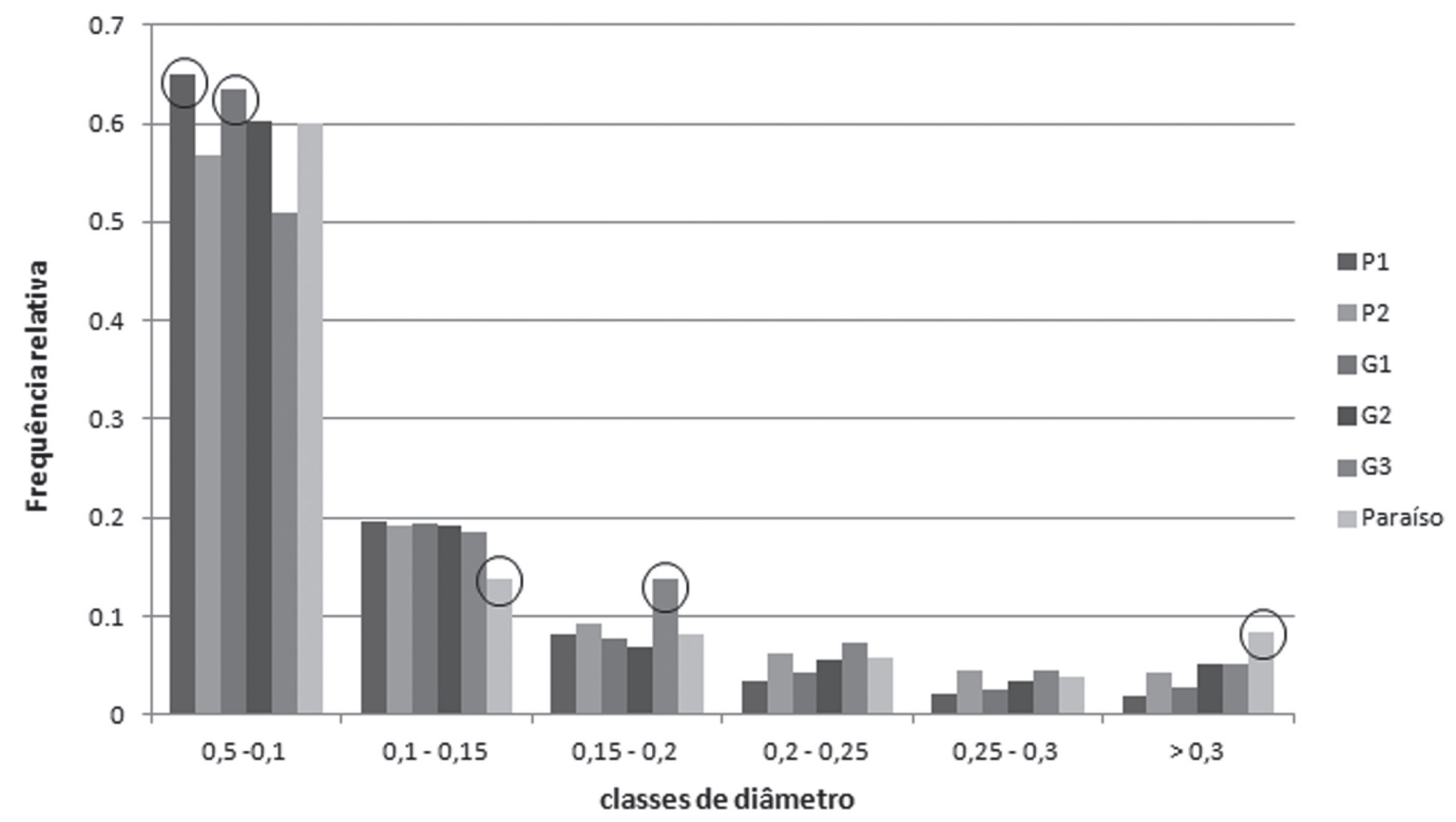

Figura 2. Proporção de indivíduos em cada classe de diâmetro para cada fragmento florestal. Os círculos indicam onde estão as diferenças significativas.

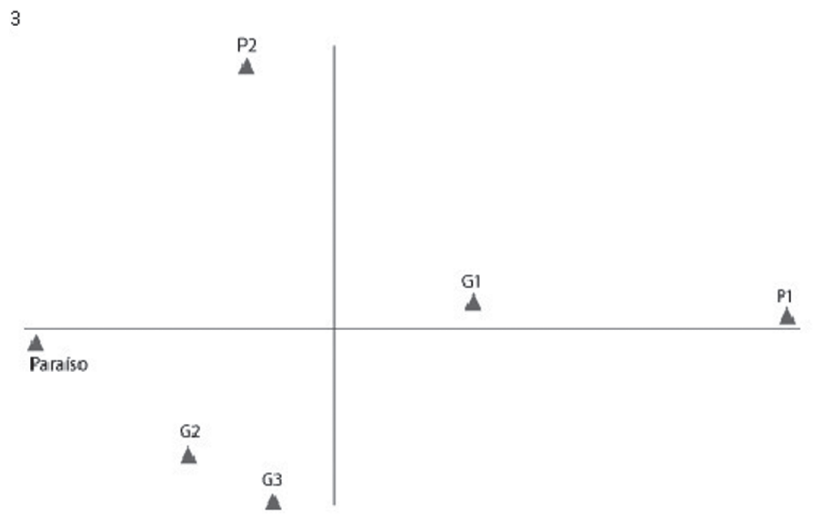

Figura 3. Posição dos fragmentos florestais em relação aos eixos 1 e 2 da análise de DCA.

que sofreram redução de área. No presente estudo, apesar de todos os fragmentos apresentarem proporções de indivíduos significativamente menores nas classes diamétricas maiores em relação à área situada em Unidade de Conservação, isto não se traduz em diferenças significativas quando da análise geral dos diâmetros. Isto está relacionado ao fato de que a existência de uma proporção maior de indivíduos em classes de diâmetro intermediárias pode diminuir as diferenças quando se consideram todas as classes agrupadas. Estudos da estrutura da vegetação em áreas consideradas de diferentes estados de conservação, em florestas tropicais da África, demonstraram que as maiores densidades e áreas basais estão presentes em comunidades consideradas de preservação moderada (Banda et al. 2006; Malaise 1984 apud Bobo et al. 2006; Zapfack et al. 2002). Maiores biomassas e riquezas foram também encontradas em sistemas florestais com tempo de regeneração intermediário em florestas tropicias da Costa Rica e do Panamá (Denslow \& Guzman 2000; Letcher \& Chazdon 2009).

Devido ao estabelecimento e crescimentos diferenciais das populações vegetais, comunidades vegetais podem formar um mosaico de estágios sucessionais, nos quais a distribuição e abundância das espécies são influenciadas ou determinadas pela dinâmica dos distúrbios naturais (Denslow 1987; Phillips \& Gentry 1994; Van der Meer et al. 1998) e antrópicos (Gascon et al. 2000; Ramirez-Marcial et al. 2001; Reyers 2004). Em florestas secundárias, este mosaico apresenta uma variabilidade da composição especifica e da riqueza, com aumento da abundância e riqueza das espécies secundárias iniciais e pioneiras (Viana et al. 1997; Villela et al. 2006; Chazdon et al. 2007, Santos et al. 2008). Todas as assembléias vegetais aqui estudadas apresentam diminuições significativas de abundância nas espécies consideradas secundárias tardias e aumento nas espécies secundárias iniciais e pioneiras quando comparadas com o sítio controle. Porém, as variações na composição específica e na estrutura de abundancias dos remanescentes estudados conferem características bastante heterogêneas ao conjunto destes remanescentes o que pode estar relacionado a tipos e intensidades de impactos diferentes a que foram submetidos.

Características como a redução de área, o isolamento dos fragmentos florestais (Turner \& Collert 1996), o tipo de matriz circundante (Mesquita et al., 1999) e a existência de distúrbios pós-fragmentação tais como a história do uso da terra, a extração seletiva de madeira e a incidência de fogo (Cochrane 2001; Holz et al. 2009) influenciam as assembléias vegetais de diferentes formas. No entanto, muitos destes dis- 
Tabela 4. Lista com o grupo sucessional (GS), Densidade Relativa (DR), Dominância Relativa (DoR) das 20 espécies de maior valor de cobertura (VC) em cada um dos fragmentos florestais. Os valores de VC podem ser encontrados somando-se DR e DoR.

\begin{tabular}{|c|c|c|c|c|c|c|c|c|c|c|c|c|c|c|}
\hline \multirow[b]{2}{*}{ FAMÍLIA } & \multirow[b]{2}{*}{ ESPÉCIE } & \multirow[b]{2}{*}{ GS } & \multicolumn{2}{|c|}{ P1 } & \multicolumn{2}{|c|}{ P2 } & \multicolumn{2}{|c|}{ G1 } & \multicolumn{2}{|c|}{ G2 } & \multicolumn{2}{|c|}{ G3 } & \multicolumn{2}{|c|}{ Paraíso } \\
\hline & & & $\mathrm{DR}$ & DoR & DR & DoR & DR & DoR & DR & DoR & DR & DoR & DR & DoR \\
\hline Anacardiaceae & Astronium graveolens Jacq. & Si & 0,00 & 0,00 & 2,11 & 0,85 & 0,00 & 0,00 & 0,27 & 2,87 & 0,00 & 0,00 & 0,00 & 0,00 \\
\hline \multirow[t]{2}{*}{ Annonaceae } & Guatteria sellowiana Schltdl & St & 0,00 & 0,00 & 0,00 & 0,00 & 2,20 & 1,77 & 0,90 & 1,61 & 0,00 & 0,00 & 0,00 & 0,00 \\
\hline & Annona sp1 & $\mathrm{Nc}$ & 0,00 & 0,00 & 0,00 & 0,00 & 0,00 & 0,00 & 0,00 & 0,00 & 0,00 & 0,00 & 0,71 & 1,87 \\
\hline \multirow[t]{2}{*}{ Apocynaceae } & $\begin{array}{l}\text { Himatanthus lancifolius (Müll.Arg.) } \\
\text { Woodson }\end{array}$ & St & 0,10 & 7,93 & 0,00 & 0,00 & 0,00 & 0,00 & 0,00 & 0,00 & 0,00 & 0,00 & 0,00 & 0,00 \\
\hline & $\begin{array}{l}\text { Malouetia cestroides (Nees ex. Mart) } \\
\text { Mull. Arg. }\end{array}$ & St & 0,00 & 0,00 & 1,38 & 1,37 & 0,00 & 0,00 & 0,00 & 0,00 & 1,08 & 2,95 & 0,00 & 0,00 \\
\hline \multirow[t]{2}{*}{ Arecaceae } & $\begin{array}{l}\text { Astrocaryum aculeatissimum (Schott) } \\
\text { Burret }\end{array}$ & $\mathrm{Pi}$ & 9,02 & 3,97 & 23,16 & 6,92 & 8,65 & 3,65 & 34,08 & 12,21 & 24,67 & 6,14 & 9,31 & 1,97 \\
\hline & Euterpe edulis Mart. & St & 0,00 & 0,00 & 0,00 & 0,00 & 0,00 & 0,00 & 0,00 & 0,00 & 4,24 & 0,97 & 1,77 & 0,29 \\
\hline Asteraceae & $\begin{array}{l}\text { Vernonanthura discolor (Spreng.) H. } \\
\text { Robb. }\end{array}$ & $\mathrm{Pi}$ & 1,96 & 3,65 & 0,00 & 0,00 & 0,00 & 0,00 & 0,00 & 0,00 & 0,00 & 0,00 & 0,00 & 0,00 \\
\hline \multirow[t]{2}{*}{ Bignoniaceae } & Jacaranda macrantha Cham. & $\mathrm{Pi}$ & 2,45 & 2,11 & 0,00 & 0,00 & 0,00 & 0,00 & 0,00 & 0,00 & 0,00 & 0,00 & 0,00 & 0,00 \\
\hline & $\begin{array}{l}\text { Handroanthus chrysotrichus (Mart. ex } \\
\text { DC.) Mattos }\end{array}$ & St & 0,00 & 0,00 & 0,00 & 0,00 & 4,04 & 1,64 & 0,00 & 0,00 & 0,00 & 0,00 & 0,00 & 0,00 \\
\hline Boraginaceae & Cordia sellowiana Cham. & $\mathrm{Si}$ & 0,00 & 0,00 & 0,00 & 0,00 & 0,00 & 0,00 & 0,00 & 0,00 & 0,50 & 7,50 & 0,00 & 0,00 \\
\hline Clethraceae & Clethra scabra Pers. & $\mathrm{Si}$ & 0,00 & 0,00 & 0,00 & 0,00 & 0,00 & 0,00 & 0,00 & 0,00 & 0,01 & 5,90 & 0,00 & 0,00 \\
\hline Clusiaceae & $\begin{array}{l}\text { Garcinia gardneriana (Planch. \& } \\
\text { Triana) Zappi }\end{array}$ & St & 0,00 & 0,00 & 0,00 & 0,00 & 0,14 & 2,79 & 0,00 & 0,00 & 0,00 & 0,00 & 0,00 & 0,00 \\
\hline Cyatheaceae & Cyathea corcovadensis (Raddi) Domin & St & 0,00 & 0,00 & 0,00 & 0,00 & 0,00 & 0,00 & 0,00 & 0,00 & 0,00 & 0,00 & 2,30 & 0,38 \\
\hline \multirow[t]{5}{*}{ Euphorbiaceae } & $\begin{array}{l}\text { Actinostemom verticillatus (Klotzsch) } \\
\text { Baill. }\end{array}$ & Nc & 0,00 & 0,00 & 1,10 & 1,01 & 0,00 & 0,00 & 0,00 & 0,00 & 0,00 & 0,00 & 0,00 & 0,00 \\
\hline & $\begin{array}{l}\text { Algernonia riedelii (Müll.Arg.) } \\
\text { G.L.Webster }\end{array}$ & $\mathrm{Si}$ & 0,00 & 0,00 & 0,00 & 0,00 & 0,00 & 0,00 & 0,00 & 0,00 & 0,00 & 0,00 & 1,77 & 1,78 \\
\hline & $\begin{array}{l}\text { Aparisthmium cordatum (A.Juss.) } \\
\text { Baill. }\end{array}$ & $\mathrm{Pi}$ & 8,43 & 4,36 & 0,37 & 2,56 & 2,20 & 1,74 & 1,98 & 7,02 & 1,99 & 1,72 & 0,00 & 0,00 \\
\hline & Mabea piriri Aubl & $\mathrm{Pi}$ & 2,16 & 2,00 & 0,00 & 0,00 & 0,00 & 0,00 & 0,00 & 0,00 & 0,00 & 0,00 & 0,00 & 0,00 \\
\hline & $\begin{array}{l}\text { Pogonophora schomburgkiana Miers } \\
\text { ex Benth }\end{array}$ & $\mathrm{Si}$ & 1,08 & 2,05 & 0,00 & 0,00 & 0,00 & 0,00 & 0,00 & 0,00 & 0,00 & 0,00 & 0,00 & 0,00 \\
\hline \multirow[t]{8}{*}{ Fabaceae } & $\begin{array}{l}\text { Albizia polycephala (Benth) Killip. } \\
\text { ex. Record }\end{array}$ & $\mathrm{Si}$ & 0,00 & 0,00 & 0,00 & 0,00 & 0,00 & 0,00 & 0,00 & 0,00 & 0,66 & 2,08 & 0,98 & 3,82 \\
\hline & Apuleia leiocarpa (Vog.) Macbr. & $\mathrm{Si}$ & 0,00 & 0,00 & 2,11 & 2,10 & 1,91 & 3,87 & 0,63 & 2,44 & 0,00 & 0,00 & 0,00 & 0,00 \\
\hline & $\begin{array}{l}\text { Chamaecrista ensiformis (Vell.) } \\
\text { H.S.Irwin \& Barneby }\end{array}$ & $\mathrm{Si}$ & 2,25 & 3,68 & 0,00 & 0,00 & 0,00 & 0,00 & 0,00 & 0,00 & 0,00 & 0,00 & 0,00 & 0,00 \\
\hline & Hymenolobium janeirense Kuhlm. & St & 0,29 & 3,06 & 0,00 & 0,00 & 0,00 & 0,00 & 0,00 & 0,00 & 0,00 & 0,00 & 0,00 & 0,00 \\
\hline & Inga lanceifolia Benth. & $\mathrm{Pi}$ & 0,98 & 2,10 & 0,00 & 0,00 & 0,00 & 0,00 & 0,00 & 0,00 & 0,00 & 0,00 & 0,00 & 0,00 \\
\hline & $\begin{array}{l}\text { Piptadenia gonoacantha (Mart) J.F. } \\
\text { Macbr. }\end{array}$ & $\mathrm{Si}$ & 0,00 & 0,00 & 3,13 & 4,28 & 0,00 & 0,00 & 0,00 & 0,00 & 0,00 & 0,00 & 0,35 & 2,67 \\
\hline & $\begin{array}{l}\text { Pseudopiptadenia contorta (DC.) G.P. } \\
\text { Lewis \& M.P. Lima }\end{array}$ & $\mathrm{Pi}$ & 0,88 & 1,96 & 1,56 & 1,93 & 0,85 & 9,06 & 0,00 & 0,00 & 0,00 & 0,00 & 0,00 & 0,00 \\
\hline & Pterocarpus rohrii Vahl. & St & 0,00 & 0,00 & 1,29 & 1,85 & 0,00 & 0,00 & 1,98 & 4,88 & 2,33 & 4,78 & 0,00 & 0,00 \\
\hline Lacistemaceae & Lacistema pubescens Mart. & $\mathrm{Si}$ & 2,84 & 1,26 & 0,00 & 0,00 & 0,00 & 0,00 & 0,00 & 0,00 & 0,00 & 0,00 & 1,95 & 0,74 \\
\hline \multirow[t]{2}{*}{ Lauraceae } & Ocotea diospirifolia (Meisn.) Mez & St & 0,00 & 0,00 & 1,56 & 7,81 & 1,49 & 3,40 & 0,00 & 0,00 & 2,41 & 4,93 & 0,00 & 0,00 \\
\hline & Ocotea sp3. & $\mathrm{Nc}$ & 1,86 & 1,03 & 0,00 & 0,00 & 0,00 & 0,00 & 1,98 & 2,37 & 0,00 & 0,00 & 0,00 & 0,00 \\
\hline Malvaceae & Pachira cf. stenopetala Casar. & St & 0,00 & 0,00 & 0,00 & 0,00 & 0,00 & 0,00 & 0,54 & 3,29 & 0,00 & 0,00 & 0,00 & 0,00 \\
\hline \multirow[t]{4}{*}{ Melastomataceae } & Miconia calvescens DC. & $P i$ & 0,00 & 0,00 & 2,57 & 0,90 & 0,00 & 0,00 & 0,00 & 0,00 & 0,00 & 0,00 & 0,00 & 0,00 \\
\hline & $\begin{array}{l}\text { Miconia cinnamomifolia (DC.) } \\
\text { Naudin }\end{array}$ & $\mathrm{Pi}$ & 7,35 & 21,06 & 0,00 & 0,00 & 0,00 & 0,00 & 0,00 & 0,00 & 0,00 & 0,00 & 0,00 & 0,00 \\
\hline & Miconia prasina (Sw.)DC. & $\mathrm{Pi}$ & 7,25 & 2,73 & 0,00 & 0,00 & 0,00 & 0,00 & 0,00 & 0,00 & 0,00 & 0,00 & 0,00 & 0,00 \\
\hline & Tibouchina granulosa (Desr.) Cogn. & $\mathrm{Pi}$ & 3,43 & 3,28 & 0,00 & 0,00 & 0,00 & 0,00 & 0,00 & 0,00 & 0,00 & 0,00 & 0,00 & 0,00 \\
\hline Meliaceae & Guarea guidonia (L.) Sleumer & $\mathrm{Si}$ & 0,00 & 0,00 & 0,00 & 0,00 & 0,00 & 0,00 & 0,81 & 1,81 & 0,00 & 0,00 & 7,09 & 18,76 \\
\hline
\end{tabular}


Tabela 4. Continuação.

\begin{tabular}{|c|c|c|c|c|c|c|c|c|c|c|c|c|c|c|}
\hline \multirow[b]{2}{*}{ FAMÍLIA } & \multirow[b]{2}{*}{ ESPÉCIE } & \multirow[b]{2}{*}{ GS } & \multicolumn{2}{|c|}{$\mathrm{P} 1$} & \multicolumn{2}{|c|}{$\mathrm{P} 2$} & \multicolumn{2}{|c|}{ G1 } & \multicolumn{2}{|c|}{ G2 } & \multicolumn{2}{|c|}{ G3 } & \multicolumn{2}{|c|}{ Paraíso } \\
\hline & & & $\mathrm{DR}$ & DoR & DR & DoR & DR & DoR & DR & DoR & DR & DoR & DR & DoR \\
\hline & Guarea macrophylla Vahl. & St & 0,00 & 0,00 & 7,54 & 10,64 & 2,70 & 5,62 & 0,63 & 1,88 & 3,82 & 5,83 & 0,89 & 2,27 \\
\hline & Cabralea canjerana (Vell.) Mart. & $S i$ & 0,00 & 0,00 & 2,94 & 2,52 & 3,05 & 4,76 & 0,00 & 0,00 & 2,16 & 2,71 & 1,51 & 4,30 \\
\hline & Cedrela odorata $\mathrm{L}$. & St & 0,00 & 0,00 & 0,00 & 0,00 & 0,00 & 0,00 & 0,00 & 0,00 & 0,00 & 0,00 & 1,42 & 1,16 \\
\hline \multirow[t]{2}{*}{ Monimiaceae } & Mollinedia aff. oligantha Perkins & St & 0,00 & 0,00 & 0,00 & 0,00 & 0,00 & 0,00 & 0,00 & 0,00 & 0,00 & 0,00 & 1,51 & 0,74 \\
\hline & Mollinedia widgrenii A.DC. & St & 0,00 & 0,00 & 1,10 & 1,11 & 0,00 & 0,00 & 0,00 & 0,00 & 0,00 & 0,00 & 0,00 & 0,00 \\
\hline \multirow[t]{4}{*}{ Moraceae } & Ficus gomelleira Kunth \& C.D.Bouché & St & 0,00 & 0,00 & 0,00 & 0,00 & 0,00 & 0,00 & 0,00 & 0,00 & 0,00 & 0,00 & 0,18 & 5,70 \\
\hline & Ficus pulchella Schott & St & 0,00 & 0,00 & 0,00 & 0,00 & 0,00 & 0,00 & 0,00 & 0,00 & 0,00 & 0,00 & 0,44 & 2,08 \\
\hline & Sorocea guilleminiana Gaudich. & $\mathrm{Si}$ & 0,00 & 0,00 & 1,93 & 3,43 & 0,00 & 0,00 & 1,98 & 1,05 & 0,00 & 0,00 & 0,00 & 0,00 \\
\hline & $\begin{array}{l}\text { Helicostylis tomentosa (Poepp. \& } \\
\text { Endl.) Rusby }\end{array}$ & $S i$ & 0,00 & 0,00 & 1,29 & 0,85 & 3,19 & 2,32 & 6,83 & 5,61 & 0,00 & 0,00 & 0,00 & 0,00 \\
\hline Myristicaceae & Myristica oleifera Schott & St & 0,00 & 0,00 & 0,00 & 0,00 & 0,00 & 0,00 & 0,00 & 0,00 & 1,25 & 1,48 & 0,00 & 0,00 \\
\hline \multirow[t]{2}{*}{ Myrtaceae } & Eugenia pisiformis Cambess. & St & 0,00 & 0,00 & 0,00 & 0,00 & 0,00 & 0,00 & 2,61 & 0,83 & 0,00 & 0,00 & 0,00 & 0,00 \\
\hline & Plinia edulis (Vell.) Sobral & St & 0,00 & 0,00 & 0,00 & 0,00 & 0,99 & 1,74 & 0,00 & 0,00 & 0,00 & 0,00 & 0,00 & 0,00 \\
\hline Nyctaginaceae & Guapira opposita (Vell.) Reitz & $\mathrm{Si}$ & 0,00 & 0,00 & 0,00 & 0,00 & 1,42 & 1,89 & 0,00 & 0,00 & 1,25 & 2,12 & 7,27 & 3,31 \\
\hline Peraceae & $\begin{array}{l}\text { Pera heteranthera (Schrank) } \\
\text { I.M.Johnst. }\end{array}$ & $\mathrm{Si}$ & 0,00 & 0,00 & 0,00 & 0,00 & 0,00 & 0,00 & 0,00 & 0,00 & 1,08 & 1,56 & 0,00 & 0,00 \\
\hline Phyllantaceae & Hieronyma oblonga (Tul.) Müll.Arg. & $S i$ & 0,00 & 0,00 & 0,00 & 0,00 & 2,13 & 2,43 & 0,00 & 0,00 & 0,00 & 0,00 & 0,00 & 0,00 \\
\hline \multirow[t]{7}{*}{ Rubiaceae } & Alseis floribunda Schott & St & 0,00 & 0,00 & 0,00 & 0,00 & 0,00 & 0,00 & 0,00 & 0,00 & 0,00 & 0,00 & 1,42 & 0,63 \\
\hline & Bathysa stipulata (Vell.) C.Presl. & St & 0,00 & 0,00 & 0,00 & 0,00 & 0,00 & 0,00 & 0,00 & 0,00 & 6,56 & 4,61 & 3,81 & 2,65 \\
\hline & $\begin{array}{l}\text { Coussarea meridionalis (Vell.) Müll. } \\
\text { Arg. }\end{array}$ & St & 0,00 & 0,00 & 0,00 & 0,00 & 0,00 & 0,00 & 3,69 & 4,35 & 0,00 & 0,00 & 0,00 & 0,00 \\
\hline & Coussarea nodosa (Benth.) Müll.Arg. & $S i$ & 0,00 & 0,00 & 0,00 & 0,00 & 7,87 & 2,77 & 0,00 & 0,00 & 0,00 & 0,00 & 0,00 & 0,00 \\
\hline & Psychotria glaziovii Müll.Arg. & $\mathrm{Si}$ & 0,00 & 0,00 & 0,00 & 0,00 & 0,00 & 0,00 & 2,43 & 2,12 & 0,00 & 0,00 & 0,00 & 0,00 \\
\hline & Psychotria hastisepala Müll.Arg. & $\mathrm{Si}$ & 0,00 & 0,00 & 0,00 & 0,00 & 0,00 & 0,00 & 1,62 & 1,57 & 0,00 & 0,00 & 0,00 & 0,00 \\
\hline & Simira glaziovii (K.Schum.) Steyerm. & $\mathrm{Si}$ & 2,25 & 1,29 & 0,00 & 0,00 & 0,00 & 0,00 & 0,00 & 0,00 & 0,00 & 0,00 & 0,00 & 0,00 \\
\hline Salicaceae & Casearia arborea (Rich.) Urb. & $\mathrm{Si}$ & 3,04 & 1,22 & 1,47 & 2,16 & 0,00 & 0,00 & 0,00 & 0,00 & 1,99 & 1,27 & 0,00 & 0,00 \\
\hline \multirow[t]{3}{*}{ Sapindaceae } & Cupania furfuracea Radlk. & $\mathrm{Si}$ & 0,00 & 0,00 & 0,00 & 0,00 & 1,70 & 1,14 & 1,26 & 2,59 & 1,58 & 1,88 & 0,00 & 0,00 \\
\hline & Cupania oblongifolia Mart. & $S i$ & 0,00 & 0,00 & 1,19 & 1,23 & 0,00 & 0,00 & 0,00 & 0,00 & 0,00 & 0,00 & 0,00 & 0,00 \\
\hline & Cupania racemosa (Vell.) Radlk. & $\mathrm{Si}$ & 3,92 & 1,29 & 0,00 & 0,00 & 2,70 & 1,12 & 2,43 & 4,47 & 0,00 & 0,00 & 0,00 & 0,00 \\
\hline \multirow[t]{2}{*}{ Sapotaceae } & Chrysophyllum flexuosum Mart. & St & 0,00 & 0,00 & 0,00 & 0,00 & 0,00 & 0,00 & 2,61 & 2,05 & 0,00 & 0,00 & 5,50 & 2,69 \\
\hline & Ecclinusa ramiflora Mart. & St & 0,00 & 0,00 & 1,29 & 1,04 & 0,00 & 0,00 & 0,00 & 0,00 & 0,00 & 0,00 & 0,00 & 0,00 \\
\hline Simaroubaceae & Picramnia glazioviana Engl. & $\mathrm{Si}$ & 0,00 & 0,00 & 0,00 & 0,00 & 0,00 & 0,00 & 0,00 & 0,00 & 0,42 & 2,24 & 0,00 & 0,00 \\
\hline \multirow[t]{2}{*}{ Siparunaceae } & Siparuna guianensis Aubl. & Si & 0,00 & 0,00 & 1,38 & 2,69 & 2,98 & 1,77 & 0,00 & 0,00 & 1,50 & 1,49 & 0,00 & 0,00 \\
\hline & Siparuna reginae Tull.A.DC. & Si & 0,00 & 0,00 & 0,00 & 0,00 & 2,62 & 2,55 & 0,00 & 0,00 & 0,00 & 0,00 & 2,39 & 1,43 \\
\hline Urticaceae & Cecropia hololeuca Miq. & $\mathrm{Pi}$ & 2,94 & 3,94 & 0,00 & 0,00 & 0,00 & 0,00 & 0,00 & 0,00 & 0,00 & 0,00 & 0,00 & 0,00 \\
\hline Violaceae & Rinorea guianensis Aubl. & $\mathrm{Si}$ & 0,00 & 0,00 & 0,00 & 0,00 & 4,18 & 2,37 & 1,26 & 1,78 & 0,00 & 0,00 & 0,00 & 0,00 \\
\hline
\end{tabular}

túrbios estão interrelacionados, atuando de forma sinérgica (Phillips 1997). Por exemplo, a incidência de fogo nas comunidades vegetais da Amazonia é considerada um efeito de borda em larga escala (Laurance 2000) e parece ser favorecida pela criação de bordas florestais (Cochrane \& Schulze 1999) e pela extração seletiva de madeira (Holdsworth \& Uhl 1997). O conjunto de caracteríticas do fragmento P1, relacionados a uma fase mais inicial de sucessão, provavelmente, resultam do conjunto de distúrbios associados a ele: este fragmento, além das evidências de existência corte seletivo em seu interior, sofreu grande redução de área e a incidência de fogo.

O fragmento G1, apesar de apresentar uma abundância maior de espécies secundárias inicias, apresenta densidade alta, uma redução significativa dos valores diamétricos e aumento nas proporções de indivíduos na classe diamétrica menor, o que indica que este fragmento apresenta características de fase inicial de regeneração secundária. $\mathrm{O}$ fato de ter havido intensa extração de 


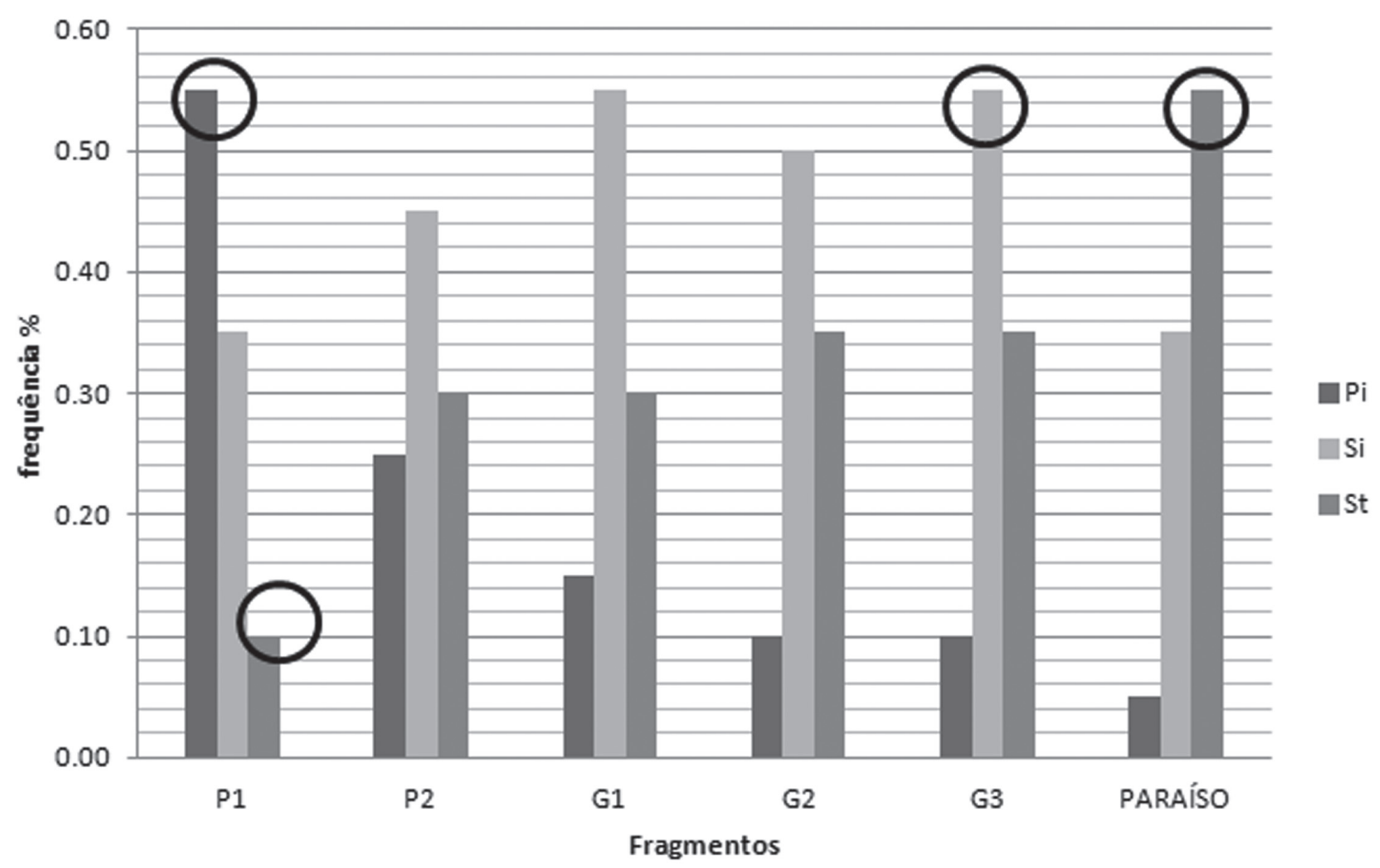

Figura 4. Proporção de espécies Pioneiras (Pi), Secundarias iniciais ( $\mathrm{Si}$ ) e Secundárias tardias (St) nos fragmentos estudados. Os círculos pretos marcam as proporções que foram significativamente diferentes em todas as comparações.

madeira no passado pode haver sido determinante para induzir essas características, já que a extração de madeira resulta na formação de clareiras e aumenta a possibilidade do estabelecimento das espécies secundárias (Holdsworth \& Uhl 1997).

A redução da área per se pode ser importante para a diminuição da riqueza de espécies e a mudança na composição específica em fragmentos florestais (Turner et al. 1996). No entanto, o fragmento de menor área deste estudo apresentou características mais próximas da área considerada como controle em comparação com outros fragmentos florestais. Sendo assim, a intensidade e frequência de outros impactos antrópicos devem também ser considerados como fatores tão determinantes quanto a perda de área e a formação de bordas florestais nas alterações da comunidade (Guariguarta \& Ostertag 2001; Tabarelli et al. 2004; Pereira et al. 2007; Latcher \& Chazdon 2009).

Desta forma, as características das comunidades vegetais podem ser resultado de ações realizadas em escalas de tempo diferentes, tanto passadas como presentes, determinando um mosaico florestal resultante de efeitos assincrônicos no espaço. Para que essas diversas escalas sejam levadas em consideração, a fim de compreender quais fatores influenciam a regeneração ou degradação florestal, é necessário, por um lado, considerar as variações naturais dos atributos locais, como as características topológicas e os tipos de solo, e, de outro, entender os processos de configuração da paisagem, ou seja, a dinâmica da fragmentação florestal e dos diferentes tipos de impactos, avaliando qualitativamente e quantitativamente os efeitos relativos de cada um desses fatores.

De maneira geral, nossos resultados sugerem que existe um aumento da degradação florestal relacionado ao tipo de propriedades do entorno: os fragmentos florestais localizados dentro de grandes propriedades rurais apresentam características sucessionais mais avançadas em relação àqueles rodeados por pequenas propriedades. A hipótese de que os remanescentes florestais rodeados por pequenas propriedades rurais estão sujeitos a tipos e intensidades de distúrbios mais intensos pode ser formulada. Entretanto, parece razoável propor que todos estes fragmentos devam ser considerados prioritários em ações de conservação dadas as suas singularidades quanto à composição específica e à riqueza.

Cabe ressaltar, ainda, que algumas assembléias vegetais em estágio de sucessão inicial apresentam-se estáveis há pelo menos 60 anos sugerindo que podem estar chegando a condições de relativo equilíbrio mais do que representar estágios sucessionais transitórios (Tabarelli et al. 1999; Santos et al. 2008). O monitoramento ao longo do tempo das modificações na estrutura e composição destas comunidades vegetais pode fornecer subsídios importantes tanto para o entendimento de seus processos ecológicos subjacentes como para a elaboração de estratégias de conservação no médio e longo prazo. 


\section{Agradecimentos}

A Jorge Caruzo, a João Henrique e Henrique Bastos pela amizade e ajuda no campo e nas diversas fases desse trabalho. Aos pesquisadores Ana Claudia Delciellos e Antonio Aisengart pela ajuda na logística e planejamento dos trabalhos de campo. Aos dois revisores anônimos que fizeram importantes contribuições para este manuscrito. Aos pesquisadores do Jardim Botânico pela ajuda na identificação das espécies, são eles: Ronaldo Marquete (Lacistemaceae e Salicaceae), Alexandre Quinet (Lauraceae), Adriana Quintella Lobão (Annonaceae), Anderson Machado (Moraceae), Genise Freire (Sapindaceae), Alessandro da Silva Rosário e Marcelo Souza (Myrtaceae), Ariane Luna Peixoto (Monimiaceae), Cyl Farney (Nyctaginaceae), Elsie Franklin Martins (Boraginaceae), Haroldo Cavalcanti e Robson Luís (Fabaceae), José Fernando Baumgratz (Melastomataceae), Luci de Senna Valle (Euphorbiaceae), Mássimo Bovini (Solanaceae). Nilda Marquete (Violaceae) e Sebastião Neto (Rubiaceae). A FAPERJ pela bolsa nota 10 e ao CNPq pela bolsa e taxa de bancada. Ao FNMA e MMA/PDA pelo financiamento desse trabalho.

\section{Referências}

Ab'Saber, A. 1971. A organização natural das paisagens inter e subtropicais brasileiras. Pp. 1-19. In: Ferri, M.G. (Org). III Simpósio sobre o Cerrado. São Paulo, Ed. Blucher/EDUSP.

Amador, E. 1997. Baia de Guanabara e Ecossistemas periféricos: Homem e natureza. Tese de Doutorado, Universidade Federal do Rio de Janeiro, Rio de Janeiro.

Antunes, V.Z.; Maia, V.C. \& Uzêda, M.C. 2009. Pré-história e história da ocupação humana. Pp. 15-31 In: Plano de Manejo APA da Bacia do Rio Macacu, Encarte 4-APA da Bacia do Rio Macacu e sua área de influência. Rio de Janeiro, Instituto BioAtlântica e PDA/MMA.

Aragão, M.B. 1961. Sobre a vegetação das zonas úmidas do Brasil. Revista Brasileira de Biologia 21 (3): 317-324.

Banda, T.; Schwartz, M.W. \& Caro, T. 2006. Woody vegetation structure and composition along a protection gradient in a miombo ecosystem of western Tanzania. Forest Ecology and Management 230: 179-185.

Bierkeland, P.W. 1974. Topography-soil relationships. In: Soil and Geomorphology. New York, Oxford University Press.

Bobo, K.S.; Waltert, M.; Sainge, N.M.; Njokagbor, J.; Fermon, H. \& Muhlenberg, M. 2006. From forest to farmland: species richness patterns of trees and understorey plants along a gradient of forest conversion in Southwestern Cameroon. Biodiversity and Conservation 15: 4097-4117.

Boher, C.B.A. 2007. Diagnóstico Ambiental das Bacias do Rio Macacu e Caceribú: Cobertura vegetal, uso e ocupação do solo. Relatório Técnico Final. Rio de Janeiro, PETROBRÂS.

Borém, R.A. \& Ramos, D.P.R. 2001. Estrutura fitossociológica da comunidade arbórea de uma topossequência pouco alterada de uma área de Floresta Atlântica, no município de Silva Jardim-RJ. Revista Árvore 25(1): 131-140.

Cabral, D.C. \& Fiszon, J.T. 2004. Padrões sócio-espaciais de desflorestamento e suas implicações para a fragmentação florestal: Estudo de caso na Bacia do Rio Macacu, RJ. Scientia Forestalis 66: 13-24.

Cabral, D.C. 2006. 'Pau-pra-toda-obra': Paisagem Florestal e Usos da Madeira na Bacia do Rio Macacu, Rio de Janeiro, final do século XVIII. Rio de Janeiro, III Encontro da ANPPAS.

Carvalho, F.A.; Nascimento, M.T. \& Braga, J.M.A. 2006. Composição e riqueza florística do componente arbóreo da Floresta Atlântica submontana na região de Imbaú, Município de Silva Jardim, RJ. Acta Botanica Brasilica 20(3): 727-740.

Carvalho, F.A.; Nascimento, M.T. \& Braga, J.M.A. 2007. Estrutura e composição florística do estrato arbóreo de um remanescente de Mata Atlântica Submontana no município de Rio Bonito, RJ, Brasil (mata do Rio Vermelho). Revista Árvore 31(4): 717-730.

Carvalho, F.A.; Nascimento, M.T. \& Oliveira Filho, A.T. 2008. Composição, riqueza e heterogeneidade da flora arbórea da bacia do rio São João, RJ, Brasil. Acta Botanica Brasilica 22(4): 929-940.

Chazdon, R.L.; Letcher, S.G.; Van Breugel, M.; Martinez-Ramos, M.; Bongers, F. \& Finegan, B. 2007. Rates of change in tree communities of secondary Neotropical forests following major disturbances. Philosophical Transactions of the Royal Society B-Biological Sciences 362: 273-289.

Cochrane, M.A. \& Schulze, M.D. 1999. Fire as recurrent event in tropical forests of the Eastern Amazon: effects on forest strucure, biomass and species composition. Biotropica 31(1): 2-16.

Cochrane, M.A. 2001. Synergistic interactions between habitat fragmentation and fire in evergreen tropical forests. Conservation Biology 15(6): 1515-1521.

Denslow, J.S. 1987. Tropical Rainforest Gaps and Tree Species Diversity. Annual Review of Ecology and Systematics 18(1): 431-451.

Denslow J.S. \& Guzman G.S. 2000. Variation in stand structure,light and seedling abundance across a tropical moist forest chronosequence, Panama. Journal of Vegetation Science 11: 201-212.

Dean, W. 2002. A Ferro e Fogo - A história e a devastação da Mata Atlântica Brasileira. 2 ed. São Paulo, Companhia das Letras.

De Walt, J.; Maliakal, S.K. \& Denslow, J.S. 2003. Changes in vegetation structure and composition along a tropical forest chornosequence: implicains for wildlife. Forest Ecology and Management 182: 139-151.

Dobson, A.P.; Bradshaw, A.D. \& Baker, A.J.M. 1997. Hopes for the Future: Restoration Ecology and Conservation Biology. Science 277: 515-522.

Drummond, J.A. 1997. Devastação e preservação ambiental no Rio de Janeiro: os parques nacionais do estado do Rio de Janeiro. Niterói - RJ, EDUFF.

Figueiredo, H.R. 1950. A flora e a fauna de Maricá. Anuário Geográfico do Estado do Rio de Janeiro 3: 13-48.

Freitas, S.R.; Mello, M.C.S. \& Cruz, C.B.M. 2005. Relationships between forest structure and vegetation indices in Atlantic Rainforest. Forest Ecology and Management 218(1-3): 353-362.

Gandolfi, S.; Leitão-Filho, H.F. \& Bezerra, C.L.F. 1995. Levantamento florístico e caráter sucessional das espécies arbustivo-arbóreas de uma floresta mesófila semidecídua no município de Guarulhos, SP. Revista Brasileira de Biologia 55: 753-767.

Gascon, C.; Williamson, B. \& Fonseca, G. 2000. Receding forest edges and vanishing reserves. Science 288: 1356-1358.

Gomes, A.P.C.; Souza, A,L. \& Neto, J.A.A.M. 2004. Alteração estrutural de uma área florestal explorada convencionalmente na bacia do Rio Paraíba do Sul, Minas Gerais, nos domínios de Floresta Atlântica. Revista Árvore 28(3): 407-417.

Guariguata, M.R. \& Ostertag, R. 2001. Neotropical secondary forest succession: changes in structural and functional characteristics. Forest Ecology and Management 148:185-206.

Guedes-Bruni, R.R. 1988. Composição florística e estrutura de um trecho de mata perturbada de baixada no município de Magé, Rio de Janeiro. Arquivos do jardim Botânico do Rio de Janeiro 29: 155-200.

Hill, M.O. \& H.G. Gauch, Jr. 1980. Detrended Correspondence Analysis: an improved ordination technique. Vegetatio 42: 47-58.

Holdsworth, A.R. \& Uhl, C. 1997. Fire in eastern Amazonian logged rain forest and the potential fire reduction. Ecological Applications 7: 713-725.

Holz, S.; Placci, G. \& Quintana, R. D. 2009. Effects of history of use on secondary forest regeneration in the Upper Parana Atlantic Forest (Misiones, Argentina). Forest Ecology and Management 258: 1629-1642.

IBGE. 1992. Manual Técnico da Vegetação Brasileira. Rio de Janeiro, IBGE.

Koeppen, W. 1948. Climatologia. México, Fondo de Cultura Econômica. Kurtz, B.C. \& Araújo, D.S.D. 2000. Composição florística e estrutura do componente arbóreo de um trecho de Mata Atlântica na Estação 
Ecológica Estadual do Paraíso, Cachoeiras de Macacu, Rio de Janeiro, Brasil. Rodriguésia 51(78/115): 69-112.

Laurance, W.F.; Delamonica, P.; Laurance, S.G.; Vasconcelos, H.L. \& Lovejoy, T.E. 2000. Rainforest fragmentation kills big trees. Nature 404: 836-1836.

Letcher, S.G. \& Chazdon, R.L. 2009. Rapid Recovery of Biomass, Species Richness, and Species Composition in a Forest Chronosequence in Northeastern Costa Rica. Biotropica 41(5): 608-617.

Mesquita, R.C.G.; Delamônica, P. \& Laurance, W.F. 1995 Effect of surrounding vegetation on edge-related tree mortality in Amazonian Forest fragments. Biological Conservation 91: 129-134.

Mueller-Dombois, D. \& Ellenberg, H. 1974. Aims and Methods of Vegetation Ecology. New York, John Willey \& Sons.

Murcia C. 1995. Edge effects in fragmented forests: Implications for conservation. Trends in Ecology and Evolution 10: 58-62.

Oliveira, R.R.; Zaú, A.S.; Lima, D.F.; Silva, M.B.R.; Vianna, M.C.; Sodré, D.O. \& Sampaio, P.D. 1995. Significado ecológico da orientação de enconstas no Maciço da Tijuca, Rio de Janeiro. Oecologia Brasiliensis VI: 523-541.

Oliveira, R.R. 2002. Ação antrópica e resultante sobre a estrutura e composição da Mata Atlântica na Ilha Grande, RJ. Rodriguésia 53(82): 33-58.

Oliveira, M.A.; Santos, A.M.M. \& Tabarelli, M. 2008. Profound impoverishment of the large-tree stand in a hyper-fragmented landscape of the Atlantic Forest. Forest Ecology and Management 256: 1910-1917.

Oliveira-filho, A.T.; Mello, J.M. \& Scolforo, J.R.S. 1997. Effects os past disturbance and edges on tree community structure and dynamics within a fragment of tropical semideciduous forest in south-eastern Brazil over a five-year period (1987-1992). Plant Ecology 131: 45-66

Oliveira-filho, A.T. \& Fontes, M.A.L. 2000. Patterns of floristic differentiation among Atlantic forests in south-eastern Brazil, and the influence of climate. Biotropica 32: 793-810.

Pellens, R.; Garay, I. \& Grandcolas, P. 2009. Biodiversity conservation and management in the brazilian atlantic forest: every fragment must be considered. Pp. 1-45. In: Rescigno, V. \& Maletta, S. (Eds.) Biodiversity Hotspots. New York, Nova Science Publishers Inc.

Pereira, I.M.; Oliveira-filho, A.T.; Botelho, S.A.; Carvalho, W.A.C.; Fontes, M.A.L.; Schiavin, I. \& Silva, A.F. 2007. Composição florística do compartimento arbóreo de cinco remanesncentes florestais do Maciço do Itatiaia, Minas Gerais e Rio de Janeiro. Rodriguésia 57(1): 103-126.

Pessoa, S.V.A. \& Oliveira, R.R. 2006. Análise estrutural da vegetação arbórea em três fragmentos florestais na Reserva Biológica de Poço das Antas, Rio de Janeiro, Brasil. Rodriguésia 57(3): 391-411.

Phillips, O.L. \& Gentry, A.H. 1994. Increasing turnover throught time in tropical forests. Science 263: 954-958.

Phillips, O.L. 1997. The changing ecology of tropical forests. Biodiversity and Conservation 6: 291-311.

Ramirez-Marcial, N.; Gonzalez-Espinosa, M. \& Williams-Linera, G. 2001. Anthropogenic disturbance and tree density in Montane Rain Forests in Chiapas, Mexico. Forest Ecology and Management 154: 311-326.

Reyers, B. 2004. Incorporating anthropogenic threats into evaluations of regional biodiversity and prioritisation of conservation areas in the Limpopo Province, South Africa. Biological Conservation 118 : 521-531.

Rodrigues, P.C. 1993. Bioestatística. Rio de Janeiro, Editora Universitária - Universidade Federal Fluminense.

Rodrigues, P.J. 2004. A Vegetação da Reserva Biológica União e os efeitos de borda na Mata Atlântica Fragmentada. Tese de Doutorado, Universidade Estadual do Norte Fluminense - UENF, Campos dos Goytácazes.

Rodrigues, P.J.; Abreu, R. \& Quinet, A. 2009. Levantamento Florístico e Fitossociológico. Pp. 45-63. In: Plano de Manejo APA da Bacia do Rio Macacu, Encarte 4-APA da Bacia do Rio Macacu e sua área de influência. Rio de Janeiro, Instituto BioAtlântica e PDA/MMA.

Santos, B.A.; Peres, C.A.; Oliveira, M.A.; Grillo, A.; Alves-Costa, C.A. \& Tabarelli, M. 2008. Drastic erosion in functional attributes of tree assemblages in Atlantic forest fragments of northeastern Brazil. Biological Conservation 141: 249-260.

Scudeller, V.V.; Martins, F.R. \& Sheperd, G.J. 2001. Distribution and abundance of arboreal species in the Atlantic ombrophilous dense forest in Southeastern Brazil. Plant Ecology 152: 185-199.

Tabarelli, M.; Mantovani, W. \& Peres C.A. 1999. Effects of habitat fragmentation on plant guild structure in the montane Atlantic forest of southeastern Brazil. Biological Conservation 91:119-127.

Tabarelli, M.; Silva, J.M.C. \& Gascon, C. 2004. Forest fragmentation, synergisms and the impoverishment of neotropical forests. Biodiversity and Conservation 13: 1419-1425.

Turner, I.M. 1996. Species loss in fragments of tropical rain forest: a review of the evidence. Journal of Applied Ecology 33: 200-219.

Turner, I.M. \& Collert, R.T. 1996. The conservation value of small, isolated fragments of lowland tropical rain forest. Trends in Ecology and Evolution 1I(8): 507.

Turner, I.M.; Chua, K.S.; Ong, J.S.Y.; Soong, B.C. \& Tan, H.T.W. 1996. A Century of Plant Species Loss from an isolated Fragment of Lowland Tropical Rain Forest. Conservation Biology 10(4): 1229-1244.

Turton, S.M. \& Freiburger, H.J. 1997. Edge and aspect effects on the Microclimate of a small tropical forest remnant on the Atherton Tableland Northeastern Australia. Pp. 45-54. In: Laurance, W.F. and Bierregard, R.O. (Eds.) Tropical Forest Remnants: Ecology, Management and conservation os fragmented comunities, USA, The University Chicago Press.

Van Der Meer, P.J.; Sterck, F.J. \& Bongers, F. 1998. Tree seedling performance in canopy gaps in a Tropical rain forest at Nouragues, French Guiana. Journal of Tropical Ecology 14: 119-137.

Viana, V.M.; Tabanez, A.A.J. \& Batista, J.L. 1997. Dynamics and restoration on Forest fragments in the Brazilian Atlantic moist forest. Pp. 351-365. In: Laurance, W.F. \& Bierregard, R.O. (Eds.) Tropical Forest Remnants: Ecology, Management and Conservation of fragmented comunities, USA, The University Chicago Press.

Villela, D.M.; Nascimento, M.T.; Aragão, L.E.O.C. \& Gama, D.M. 2006. Effect of selective logging on forest structure and nutrient cycling in a seasonally dry Brazilian Atlantic forest. Journal of Biogeography 33: 506-516. 